\title{
Muon Cooling in a Quadrupole Magnet Channel
}

\author{
David Neuffer, Fermilab
}

A. Poklonskiy, MSU

\begin{abstract}
As discussed before, [1] a cooling channel using quadrupole magnets in a FODO transport channel can be used for initial cooling of muons. In the present note we discuss this possibility of a FODO focusing channel for cooling, and we present ICOOL simulations of muon cooling within a FODO channel. We explore a $1.5 \mathrm{~m}$ cell-length cooling channel that could be used for the initial transverse cooling stage of a muon collider or neutrino factory.
\end{abstract}

\section{Introduction}

Many of our initial studies have used solenoidal focusing for the capture of low-energy pions from a target, and cooling of the muons, primarily because solenoids focus in both $\mathrm{x}$ and $\mathrm{y}$ and are reasonably effective in obtaining small $\beta_{\perp}$. However initial cooling does not need very small $\beta_{\perp}$. C. Johnstone et al. have suggested that initial muon capture and ionization cooling could occur within a short-period FODO quad lattice, and calculated an example in COSY.[1]

The study 2A [2] cooling and transport had a $\beta_{\perp}$ of $\sim 0.8 \mathrm{~m}$, obtained from $0.75 \mathrm{~m}$ long solenoidal cells. We initially consider a $0.75 \mathrm{~m}$ (half-cell) FODO quad channel as an alternative. This lattice has a $\beta_{\mathrm{x}}$ and $\beta_{\mathrm{y}}$ that both oscillate between $\sim 2.5 \mathrm{~m}$ and $\sim 0.9 \mathrm{~m}$, with an average of $\sim 1.75$. The lattice does not focus simultaneously in $\mathrm{x}$ and $\mathrm{y}$, so only an average value of $\beta_{\perp}$ can be used for cooling. The $\beta_{\perp}$ is sufficiently small for initial cooling, particularly if the material is a low-scattering material such as $\mathrm{H}_{2}$.

Muon cooling within a beam transport can be estimated using the rms cooling equation.[3] The cooling method used is ionization cooling, which is limited by the beam focusing and multiple scattering in the cooling absorbers. The basic cooling equation for transverse muon cooling within energy loss absorbers is:

$$
\frac{\mathrm{d} \varepsilon_{\mathrm{N}}}{\mathrm{ds}}=-\frac{\mathrm{g}_{\mathrm{t}}}{\mathrm{P}_{\mu}} \frac{\mathrm{dP}}{\mathrm{ds}} \varepsilon_{\mathrm{N}}+\frac{\beta_{\perp} \mathrm{E}_{\mathrm{s}}^{2}}{2 \beta^{2} \mathrm{~m}_{\mu} \mathrm{c}^{3} \mathrm{~L}_{\mathrm{R}} \mathrm{P}_{\mu}},
$$

where $\varepsilon_{N}$ is the transverse normalized emittance, $g_{t}$ is the transverse partition number ( $\mathrm{g}_{\mathrm{t}}=1$ without emittance exchange), $\beta=\mathrm{v} / \mathrm{c}, \mathrm{P}_{\mu}$ is the muon momentum, $\mathrm{dP}_{\mu} / \mathrm{ds}$ is the momentum loss in the absorber, $\mathrm{E}_{\mathrm{s}}$ is the characteristic scattering energy $(\sim 14 \mathrm{MeV}), \mathrm{L}_{\mathrm{R}}$ is the characteristic radiation length in the material, and $\beta \perp$ is the transverse focusing function at the absorber. Cooling is limited to the equilibrium transverse emittance, which is: 


$$
\varepsilon_{\mathrm{N}, \mathrm{eq}}=\frac{\beta_{\perp} E_{\mathrm{s}}^{2}}{2 \mathrm{~g}_{\mathrm{t}} \beta \mathrm{m}_{\mu} \mathrm{c}^{2} \mathrm{~L}_{\mathrm{R}} \frac{\mathrm{dE}}{\mathrm{ds}}},
$$

where $\mathrm{dE} / \mathrm{ds}=\beta \mathrm{c} \mathrm{dP} \mathrm{P}_{\mu} / \mathrm{ds}$. $\mathrm{dE} / \mathrm{ds}$ is approximately given by the Bethe-Bloch formula :

$$
\frac{\mathrm{dE}}{\mathrm{ds}} \cong 4 \pi \mathrm{N}_{\mathrm{A}} \rho \mathrm{r}_{\mathrm{e}}^{2} \mathrm{~m}_{\mathrm{e}} \mathrm{c}^{2} \frac{\mathrm{Z}}{\mathrm{A}}\left[\frac{1}{\beta^{2}} \ln \left(\frac{2 \mathrm{~m}_{\mathrm{e}} \mathrm{c}^{2} \gamma^{2} \beta^{2}}{\mathrm{I}(\mathrm{Z})}\right)-1-\frac{\delta}{2 \beta^{2}}\right]
$$

where $\mathrm{N}_{\mathrm{A}}$ is Avogadro's number, $\mathrm{Z}, \mathrm{A}$ are the material atomic number and weight, $\rho$ is the material density and $\delta \approx 0$ is the density effect parameter $(\delta=\sim 0)$. The material with largest $\mathrm{L}_{\mathrm{R}} \mathrm{dE} / \mathrm{ds}$ is hydrogen, and therefore the best cooling material. $\mathrm{I}(\mathrm{Z})$ is the material ionization energy, and is approximately $16 \mathrm{Z}^{0.9} \mathrm{eV}$.

The parameters in equation [2] are functions of the cooling material, and the focusing parameters $\beta_{\perp}$, and the beam speed $\beta$, with $\beta \cong 1$ for For a hydrogen absorber the value (at $\left.\mathrm{g}_{\mathrm{t}}=1\right)$ is $\varepsilon_{\mathrm{N}, \mathrm{eq}}=\sim 0.0037 \beta_{\perp}$ and for $\mathrm{LiH}$ it is $\sim 0.0068 \beta_{\perp}$.

In the Neutrino factory study, a solenoidal focusing transport with a period of $0.75 \mathrm{~m}$ was used for initial cooling of the muons. For initial focusing a relatively weak focusing was used; the average $\beta_{\perp}$ was $\sim 0.8 \mathrm{~m}$, and the Study $2 \mathrm{~A}$ study had $\sim 100 \mathrm{~m}$ of such cells.

Solenoidal focusing imposes helical motion upon the particle orbits. This additional motion places a momentum-amplitude correlation for synchronous motion, complicating matching of the beam within rf accelerating systems. Solenoidal channels have losses due to mismatch of the momentum-amplitude correlation, and additional resonance from the longitudinal/transverse coupling.

Quadrupole focusing has transverse and longitudinal motion more clearly separated than with solenoids, and amplitude-momentum correlations do not limit the acceptance. Using quadrupoles for focusing requires alternating quad signs along the transport. A simplest configuration for a focusing quad transport is the FODO lattice, which consists of a sequence of cells with an $\mathrm{F}$ quad, an $\mathrm{O}$ drift, an opposite sign $\mathrm{D}$ quad and another drift. A FODO transport has a relatively large momentum acceptance; all particles with momentum greater than a small-momentum can be stably transported.

The disadvantage of the FODO transport is that it does not have a focus point with small $\beta$-function in both $\mathrm{x}$ and $\mathrm{y}$. There is therefore no preferred absorber location with minimal emittance growth from scattering in both $\mathrm{x}$ and $\mathrm{y}$. However that also means that the absorber could be diffused throughout the transport without a cooling penalty, as could be done with gaseous hydrogen.

In a quadrupole focusing system with bending magnets, a horizontal dispersion is generated. That dispersion, with wedge absorbers, can be used to obtain emittance exchange cooling, coupling the $\mathrm{x}$-motion and the longitudinal motion, with the y-motion and cooling unchanged. Dispersion with wedges can also be used for emittance exchange 
cooling within solenoids, but the $\mathrm{x}$ and $\mathrm{y}$ motions are so tightly coupled that separating $\mathrm{x}$ and y exchange is not practical. (The coupling may or may not be desirable.)

\section{Sample parameters}

As an initial example, we consider a modified version of the lattice used in the study $2 \mathrm{~A}$ cooling channel. That lattice was an alternating solenoid lattice with a period of $0.75 \mathrm{~m}$. The $0.75 \mathrm{~m}$ lattice included $0.5 \mathrm{~m}$ of rf cavity plus $21 \mathrm{~cm} \mathrm{LiH}$ absorbers, with the remaining space allowing room for focusing solenoidal coils. The betatron function within that lattice was $\sim 0.8 \mathrm{~m}$, with only a weak variation along the cell length.

For the FODO lattice we choose a half-cell period of $0.75 \mathrm{~m}$ with a quad length of $0.23 \mathrm{~m}$; the remaining $0.52 \mathrm{~m}$ is reserved for absorbers and RF. Each half-cell contains two 200.7 $\mathrm{MHz}$ pillbox $\mathrm{rf}$ cavities with $0.25 \mathrm{~m}$ length operating at $15.25 \mathrm{MV} / \mathrm{m}$ gradient. The reference momentum for the neutrino factory cooling channel is $215 \mathrm{MeV} / \mathrm{c}$. If we set the phase advance per cell at $60^{\circ}$, the required gradient is $4.15 \mathrm{~T} / \mathrm{m}$, which implies a field of $1.66 \mathrm{~T}$ at a reference radius of $0.4 \mathrm{~m}$. This field could be reached with normal-conducting ferric magnets as well as superconducting magnets. The maximum betatron function is $\sim 2.6 \mathrm{~m}$ and the minimum is $\sim 0.9 \mathrm{~m}$, with an average value of $\sim 1.6 \mathrm{~m}$. This is significantly larger than in the solenoidal channels.

The $\mu$ beam emittance from the target and capture transport is $\sim 0.02 \mathrm{~m}$ (rms, normalized). For simulation studies we initially placed a beam with these rms emittances at the start of the cooling transport. We consider two cases: one in which the absorbers were $1 \mathrm{~cm} \mathrm{LiH}$ absorbers, placed as in the Study $2 \mathrm{~A}$ report ( 2 per $0.75 \mathrm{~m}$ half-cell), and the second, in which the absorber is gaseous hydrogen which fills the beam transport at a density corresponding to $133 \mathrm{~atm}$ at $293^{\circ} \mathrm{K}$. The average energy rate loss is the same for both cases ( 3.5MeV / 0.75m transport).

Simulations of muon cooling were run using the ICOOL code,[4] with a $97.5 \mathrm{~m}$ long cooling channel $\left(65\right.$ cells). For the $\mathrm{LiH}$ absorbers, the transverse emittance $\varepsilon_{\mathrm{t}, \mathrm{N}}$ cools from $\sim 0.0204 \mathrm{~m}$ to $0.015 \mathrm{~m}$ in $\sim 25 \mathrm{~m}$, and cools to $\sim 0.012 \mathrm{~m}$ in $\sim 50 \mathrm{~m}$. The asymptotic cooled emittance is $\sim 0.010 \mathrm{~m}$. These results were obtained using the newer ICOOL scattering model based on Fano (ICOOL model 6).[5] With the older Bethe model (ICOOL model 4) the initial cooling rate is the same but the asymptotic value is $\sim 0.0115 \mathrm{~m}$, which is somewhat larger.

With hydrogen gas-filled transport, the transverse emittance $\varepsilon_{\mathrm{t}, \mathrm{N}}$ cools from $\sim 0.0204 \mathrm{~m}$ to $0.015 \mathrm{~m}$ in $\sim 20 \mathrm{~m}$, and cools to $\sim 0.010 \mathrm{~m}$ in $\sim 50 \mathrm{~m}$, and is cooled further to $\sim 0.0065 \mathrm{~m}$ at $\sim 100 \mathrm{~m}$. The asymptotic cooled emittance is $\sim 0.005 \mathrm{~m}$. The difference between scattering models 4 and 6 was small, much less than in the $\mathrm{LiH}$ simulations. The hydrogen-filled gas absorber had performance similar to the Study 2A cooling channel solenoid case with $\mathrm{LiH}$ absorbers, indicating that the quad channel could be used as a substitute for that cooling channel, with similar potential performance. The cooling with gas absorbers is somewhat better than the simple rms formula; the equilibrium emittance is $\sim 30 \%$ less 
than a simple insertion of the values would indicate. Figure 2 shows these changes in emittance along the cooling channel for the cases of $\mathrm{LiH}$ absorbers and $\mathrm{H}_{2}$ gas absorbers.

Unlike solenoidal channels there is no large amplitude-energy correlation, and a much reduced problem of beam loss in matching and transport through the channel because of that correlation.

A significant advantage of the quad channel is that the magnetic field in the rf cavities is much weaker and is tranverse rather than parallel to the accelerating field. Initial MTA observations indicate that solenoidal fields may limit the peak gradient achievable in pillbox cavities, and it is likely that the weaker transverse fields may not limit the gradients.[6] Initial experiments with pillbox cavities within magnetic fields indicate a large reduction in possible gradient because of this effect.

\section{Other Examples}

Our baseline example corresponded to a phase advance of $60^{\circ} /$ cell at $215 \mathrm{MeV} / \mathrm{c}$. We also considered a case with $90 \%$ cell, obtained by increasing the quad gradient to 5.88 $\mathrm{T} / \mathrm{m}$. This focuses the beam to a smaller average $\beta_{\perp}$, although the peak value remains the same. In ICOOL simulations this enables cooling to smaller emittances ( 20\%), but the acceptance of the channel is slightly reduced.

We have made a first attempt at incorporating the quad channel into the study $2 \mathrm{~A}$ channel. We replace the solenoidal cooling channel with the FODO cooling channel. A 4-quad match from constant $\beta_{\perp}=0.8 \mathrm{~m}$ into the quad channel was generated using DIMAD. (total length of $1.935 \mathrm{~m}$ ) This matches the linear lattice, but does not fully match the transition from solenoidal optics (with amplitude-energy correlations) into separated function FODO optics. The buncher and phase-rotator of Study 2A (with solenoidal B= $1.75 \mathrm{~T}$ optics) was used and the match into the FODO cooler inserted at the end of the phase rotator.

Initial simulations showed that beam was still somewhat mismatched, with the beam somewhat blown up in size after transfer into the FODO cooling channel. The beam then cools in a manner similar to the Study $2 \mathrm{~A}$ solenoid channel (with $\mathrm{LiH}$ absorbers.) The transverse emittance was reduced from $0.0163 \mathrm{~m}$ to $0.0065 \mathrm{~m}$ over $100 \mathrm{~m}$ (compared to 0.0060 for the solenoid case), and the muons within the ECALC9[7] acceptance increased from $0.103 \mu / \mathrm{p}$ to 0.21 over the channel (compared to the increase from 0.126 $\mu / \mathrm{p}$ to 0.248 with solenoids). The performance seems a bit inferior to the Study $2 \mathrm{~A}$ case, with $\sim 10 \%$ less beam within the baseline ECALC9 acceptance than the Study 2A case. However the ECALC9 criteria are adapted to cylindrically symmetric geometries with solenoidal focusing; a more appropriate adaptation to FODO optics may modify this assessment.

We therefore rewrote the ECALC9 program as ECALC9xy[8], where $\mathrm{x}$ and $\mathrm{y}$ emittances and betatron functions are separately calculated. The acceptance criteria are then rewritten as $A_{x}+A_{y}<\varepsilon_{\text {ref }}$, where $A_{x}$ and $A_{y}$ are the separate $x$ and $y$ amplitudes, and $\varepsilon_{\text {ref }}$ 
is the reference amplitude. ECALC9 uses the criteria $2 A_{R}<\varepsilon_{\text {ref }}$, where $A_{R}$ is the radially symmetric amplitude. The two criteria obtain roughly the same acceptances for the solenoidal channel, but produces more favorable acceptances in the quad channel evaluations. The muons within the ECALC9xy acceptance increased from $0.108 \mu / \mathrm{p}$ to 0.264 over the quad channel (compared to an increase from $0.121 \mu / \mathrm{p}$ to 0.258 with solenoids). Under this evaluation, the quad channel is as effective in cooling and beam acceptance as the reference solenoid channel.

The ICOOL simulations indicate the match from the rotator into the FODO cooler is not fully optimized, with some beam blowup occuring at transition. Future optimization may improve the match and reduce losses from mismatch. This study does demonstrate that a FODO channel could be used in initial cooling of muons for a Neutrino Factory, particularly if $\mathrm{H}_{2}$ absorbers can be used. The FODO channel has some potential advantages in this cooling region over the solenoidal systems, particularly in cost and rf field compatibility.

\section{Use in Buncher and $\phi$-E Rotation}

We can also consider using quadrupole focusing in the phase energy and rotation section of the Neutrino Factory. In an initial attempt we transferred the solenoid to quad matching section to the front of the bunching section of the Study 2a example and continued quadrupole focusing through the entire bunching, phase-energy rotation, and cooling sections. The results were disappointing. About half of the muons were lost in the first few meters, and that loss of a factor of $\sim 2$ remained through the system. Most of these were lower-energy muons that apparently were outsider the transverse acceptance. Other simulations indicate that the quad channel does not accept lower-momentum muons.

A preliminary conclusion is that the quad channel does not have a large enough momentum acceptance for this section, even though it has an adequate momentum acceptance for the cooling section. This is not unexpected. The momentum acceptance requirements for the cooling channel are $\sim \pm 25 \%$; the requirements for the buncher/rotator are closer to $\pm 100 \%$.

Further studies should more accurately determine these requirements and whether a quad channel can be redesigned to match these. Further studies could also reoptimize the matching and cooling section parameters for better performance.

\section{References}

[1] C. Johnstone, M. Berz, D. Errede, K. Makino, Nucl. Inst. and Meth. A 519, 472 (2004).

[2] J. S. Berg, "Cost-effective design for a Neutrino Factory", PRSTAB 9, 011001 (2006). 
[3] D. Neuffer, Nucl., Inst. and Meth. A532, p.26 (2004).

[4] R. Fernow, "ICOOL, a Simulation Code for Ionization Cooling of Muon Beams", Proc. 1999 Particle Accelerator Conference, New York, p. 3020 (1999), see http://pubweb.bnl.gov/people/fernow/.

[5] R. Fernow, "Scattering in ICOOL", MuCOOL Note 336, April 2006.

[6] J. Norem et al., "Dark Current, breakdown and magnetic field effects in a multicell 805 MHz Cavity”, PRSTAB 6, 072001 (2003).

[7] R. Fernow, "Physics Analysis performed by ECALC9", MuCOOL Note 280, September 2003.

[8] D. Neuffer, "ECALC9xy: an adaptation of ECALC9 to non-radially symmetric motion", unpublished (2007). 
Figure 1 sketch of $1.5 \mathrm{~m}$ FODO transport cell $\left(0.75 \mathrm{~m}\right.$ half-cell), filled with $\mathrm{H}_{2}$ gas. Components include $\mathrm{F}$ and $\mathrm{D}$ quads and a $0.5 \mathrm{~m}$ region for rf cavities. (The $0.5 \mathrm{~m}$ length was split into 2 cavities in the simulations.) (Graph shows a radial cross section with $\mathrm{r}=0$ at the bottom.)

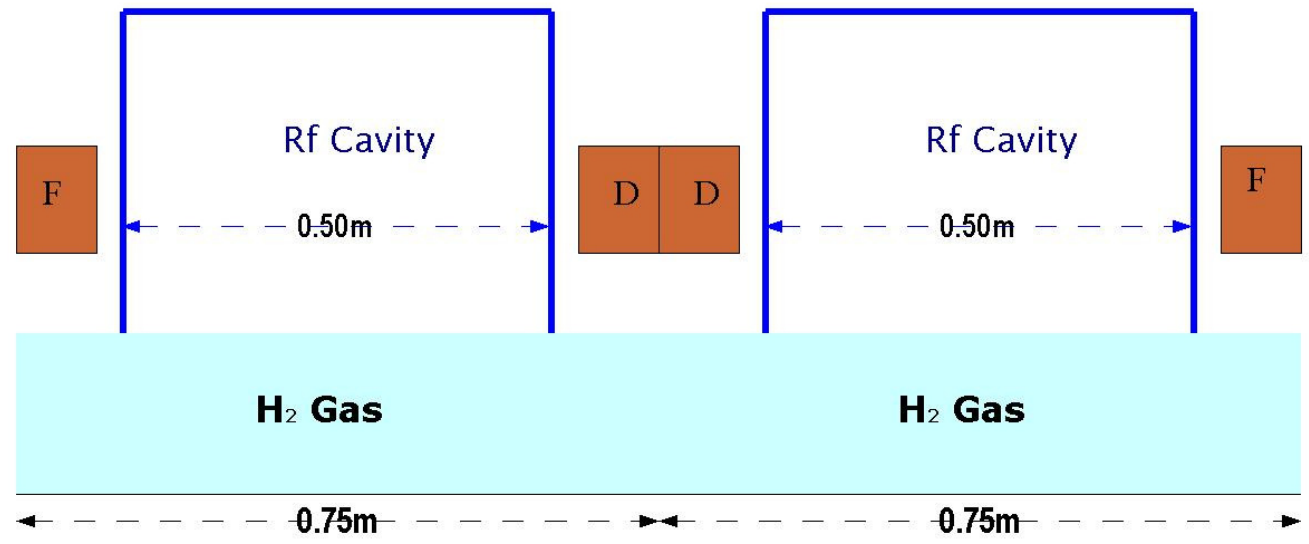

Figure 2. simulation of transverse emittance cooling in a FODO channel with $\mathrm{LiH}$ or $\mathrm{H}_{2}$ absorbers. Vertical scale is distance along the cooling channel (in $\mathrm{m}$ ) and horizontal scale is rms transverse emittance (normalized) in $\mathrm{m}$.

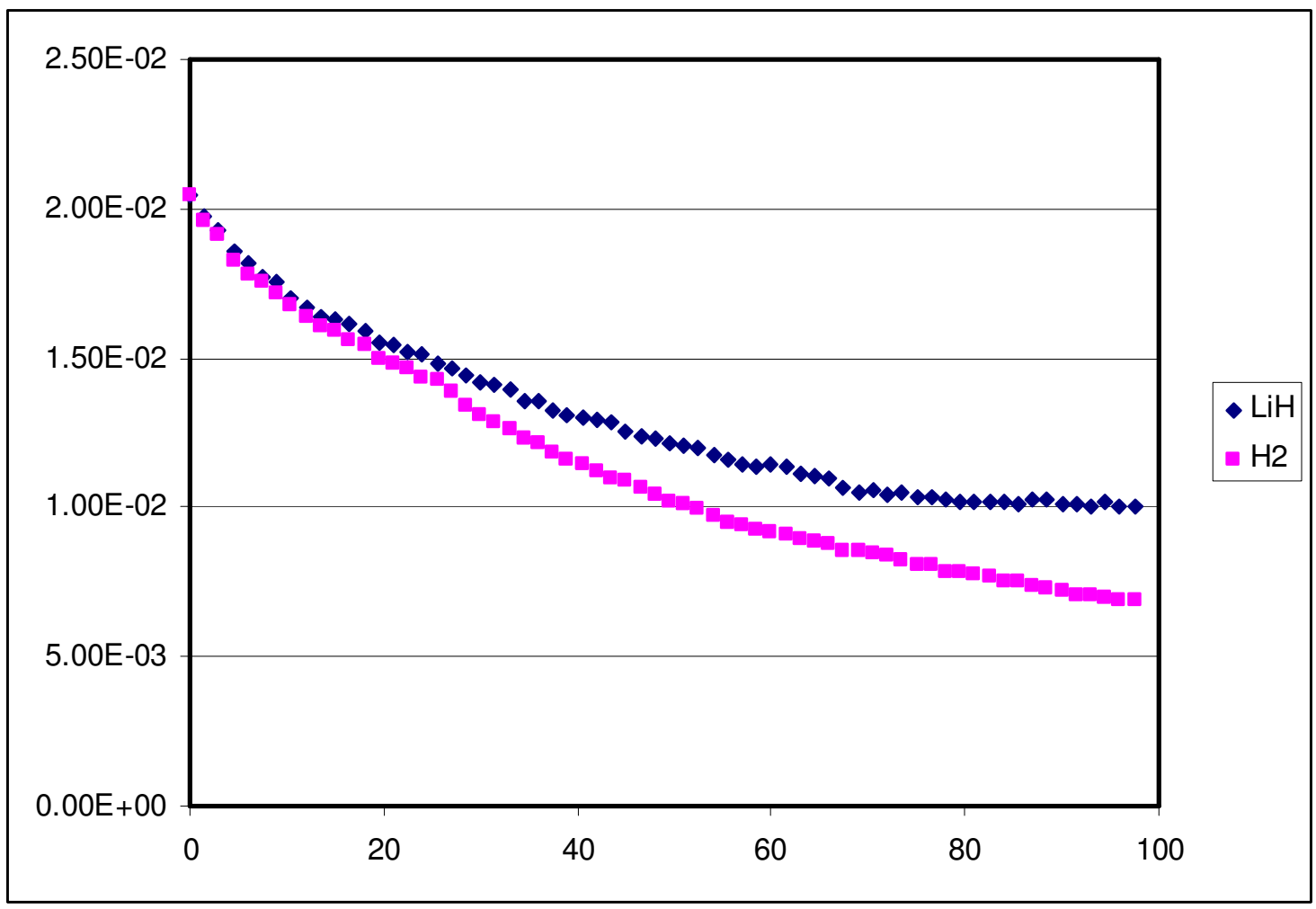


Figure 3. Muon acceptance for criteria under ECALC9xy. Simulation had 5000 initial pions and results are normalized to pions from 5000 initial $24 \mathrm{GeV}$ protons.

n0-Black line - all surviving muons.

$\mathrm{n} 2$-Blue line - muons within Study $2 \mathrm{~A}$ acceptance $\left(\mathrm{A}_{\mathrm{x}}+\mathrm{A}_{\mathrm{y}}<0.03 \mathrm{~m}-\mathrm{R}\right)$.

$n 1-P i n k$ line- muons within Study 2 acceptance $\left(A_{x}+A_{y}<0.015 m-R\right)$.

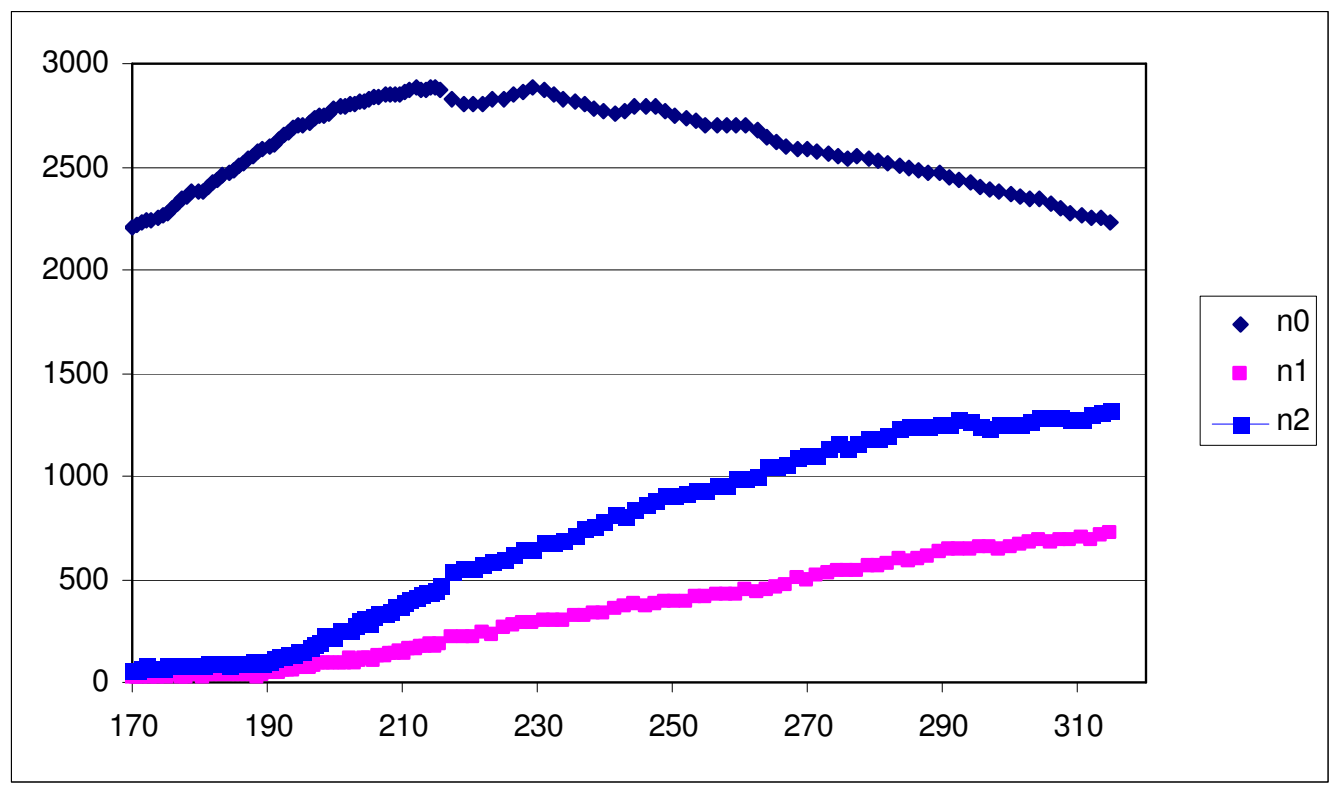

